\title{
Corrigendum: Lower Masticatory Performance Is a Risk for the Development of the Metabolic Syndrome: The Suita Study
}

\begin{abstract}
Shuri Fushida ${ }^{1}$, Takayuki Kosaka ${ }^{1 *}$, Michikazu Nakai ${ }^{2}$, Momoyo Kida $^{1}$, Takashi Nokubi ${ }^{3}$, Yoshihiro Kokubo ${ }^{4}$, Makoto Watanabe ${ }^{4}$, Yoshihiro Miyamoto ${ }^{5}$, Takahiro Ono ${ }^{1,6}$ and Kazunori lkebe ${ }^{1}$

${ }^{1}$ Department of Prosthodontics, Gerodontology and Oral Rehabilitation, Osaka University Graduate School of Dentistry, Suita, Japan, ${ }^{2}$ Center for Cerebral and Cardiovascular Disease Information, National Cerebral and Cardiovascular Center, Suita, Japan, ${ }^{3}$ Osaka University, Suita, Japan, ${ }^{4}$ Department of Preventive Cardiology, National Cerebral and Cardiovascular Center, Suita, Japan, ${ }^{5}$ Open Innovation Center, National Cerebral and Cardiovascular Center, Suita, Japan, ${ }^{6}$ Division of Comprehensive Prosthodontics, Faculty of Dentistry \& Graduate School of Medical and Dental Sciences, Niigata University, Niigata, Japan
\end{abstract}

Keywords: geriatric dentistry, prosthodontics, mastication, epidemiology, preventive dentistry, cardiovascular diseases

\section{OPEN ACCESS}

Approved by:

Frontiers Editorial Office,

Frontiers Media SA, Switzerland

*Correspondence:

Takayuki Kosaka

kosaka@dent.osaka-u.ac.jp

Specialty section:

This article was submitted to Cardiovascular Epidemiology and

Prevention,

a section of the journal

Frontiers in Cardiovascular Medicine

Received: 09 February 2022

Accepted: 10 February 2022

Published: 03 March 2022

Citation:

Fushida S, Kosaka T, Nakai M,

Kida M, Nokubi T, Kokubo Y,

Watanabe M, Miyamoto $Y$, Ono $T$ and Ikebe K (2022) Corrigendum: Lower Masticatory Performance Is a Risk for the Development of the Metabolic

Syndrome: The Suita Study.

Front. Cardiovasc. Med. 9:872326

doi: 10.3389/fcvm.2022.872326

\section{A Corrigendum on}

Lower Masticatory Performance Is a Risk for the Development of the Metabolic Syndrome: The Suita Study

by Fushida, S., Kosaka, T., Nakai, M., Kida, M., Nokubi, T., Kokubo, Y., Watanabe, M., Miyamoto, Y., Ono, T., and Ikebe, K. (2021). Front. Cardiovasc. Med. 8:752667. doi: 10.3389/fcvm.2021.752667

In the original article, the Funding statement is missing the grant number: 19K19123. The corrected statement appears below.

Funding:

"This study was supported by grants-in-aid from the Ministry of Education, Culture, Sports, Science and Technology of Japan (Nos. 20390489, 23390441, 26293411, 17H04388, and 19K19123) and internal research grants from the National Cerebral and Cardiovascular Center (22-4-5 and 27-4-3)."

The authors apologize for this error and state that this does not change the scientific conclusions of the article in any way. The original article has been updated.

Publisher's Note: All claims expressed in this article are solely those of the authors and do not necessarily represent those of their affiliated organizations, or those of the publisher, the editors and the reviewers. Any product that may be evaluated in this article, or claim that may be made by its manufacturer, is not guaranteed or endorsed by the publisher.

Copyright $\odot 2022$ Fushida, Kosaka, Nakai, Kida, Nokubi, Kokubo, Watanabe, Miyamoto, Ono and Ikebe. This is an open-access article distributed under the terms of the Creative Commons Attribution License (CC BY). The use, distribution or reproduction in other forums is permitted, provided the original author(s) and the copyright owner(s) are credited and that the original publication in this journal is cited, in accordance with accepted academic practice. No use, distribution or reproduction is permitted which does not comply with these terms. 